\title{
What is the filling defects of pulmonary trunk: a rare case of pulmonary leiomyosarcoma
}

\author{
Yun Tang ${ }^{1}$, He Jialin ${ }^{2}$, Fei Zhao ${ }^{1}$, Bo Zheng ${ }^{1}$, Shihai Tang $^{1}$, Juan Gong ${ }^{1}$, Li Wang ${ }^{1}$, and \\ Yang Zhou ${ }^{2}$ \\ ${ }^{1}$ People's Hospital of Leshan \\ ${ }^{2}$ People's Hospital of Leshan
}

September 21, 2020

\begin{abstract}
Background: Leiomyosarcoma occurs commonly in the abdomen, retroperitoneum, large blood vessels, and uterus[1]. Cardiac leiomyosarcoma is a rare and highly aggressive sarcoma. Methods and Results: Here, we report the case of a 63-year-old male with pulmonary artery leiomyosarcoma. Transthoracic echocardiography showed a large $4.4 \times 2.3 \mathrm{~cm}$ hypoechoic mass in right ventricular outflow tract and pulmonary artery. Pulmonary computed tomography angiography showed a filling defect in similar location. Surgery was performed due to tend to occlusion. A yellow mass adhered to ventricular septum and pulmonary artery wall was detected and compressed pulmonary valve. By immunohistochemistry, the tumor cells stained positive for Desmin and smooth muscle actin, and stained negative for S-100, CD34, myogenin, or myoglobin, KI67(+)80\%, supported leiomyosarcoma. Conclusion: The patient had recovered from surgery and had been on following-up.
\end{abstract}

\section{Hosted file}

manuscipt2.pdf available at https://authorea.com/users/360694/articles/482271-what-is-thefilling-defects-of-pulmonary-trunk-a-rare-case-of-pulmonary-leiomyosarcoma 

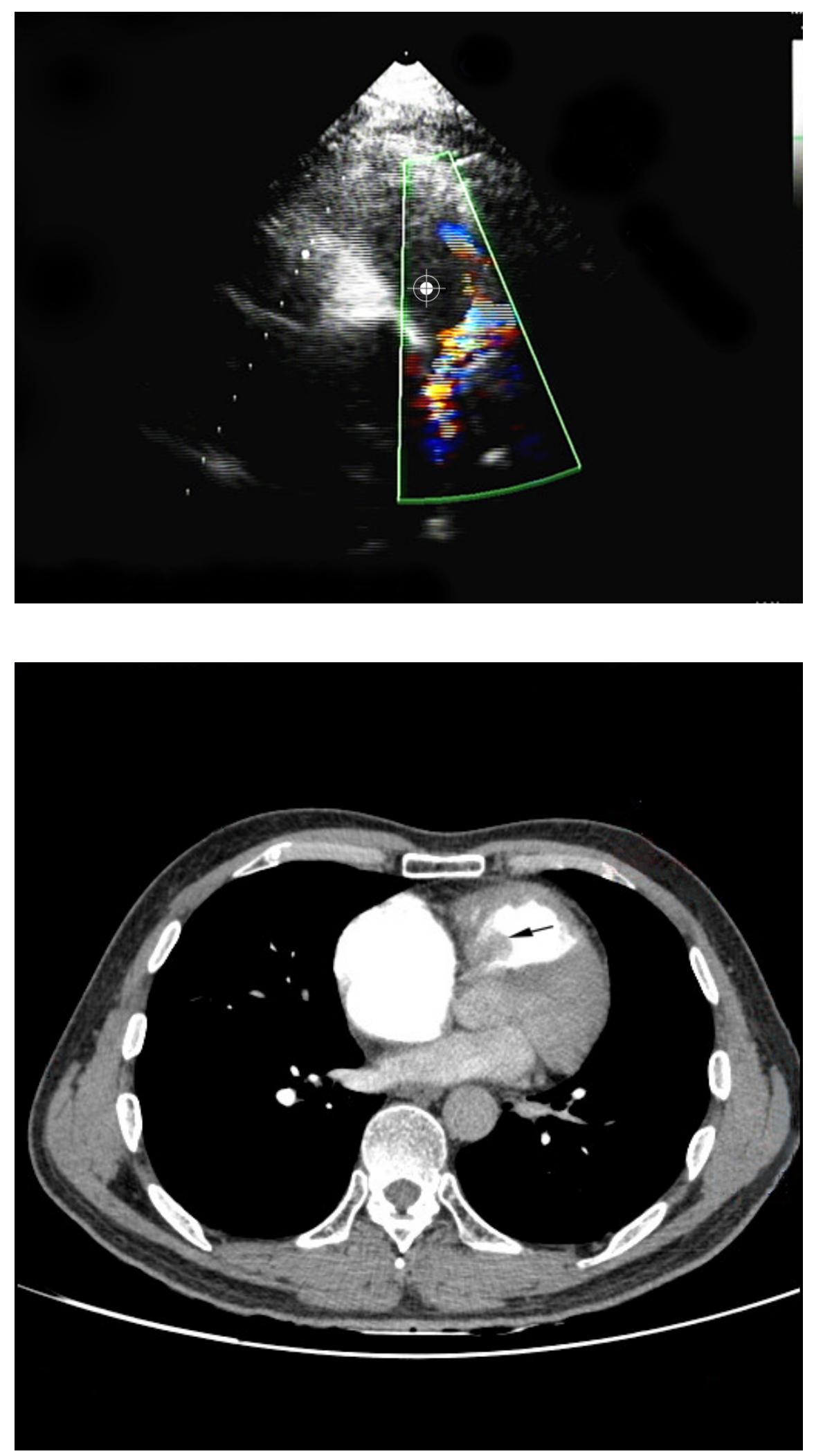


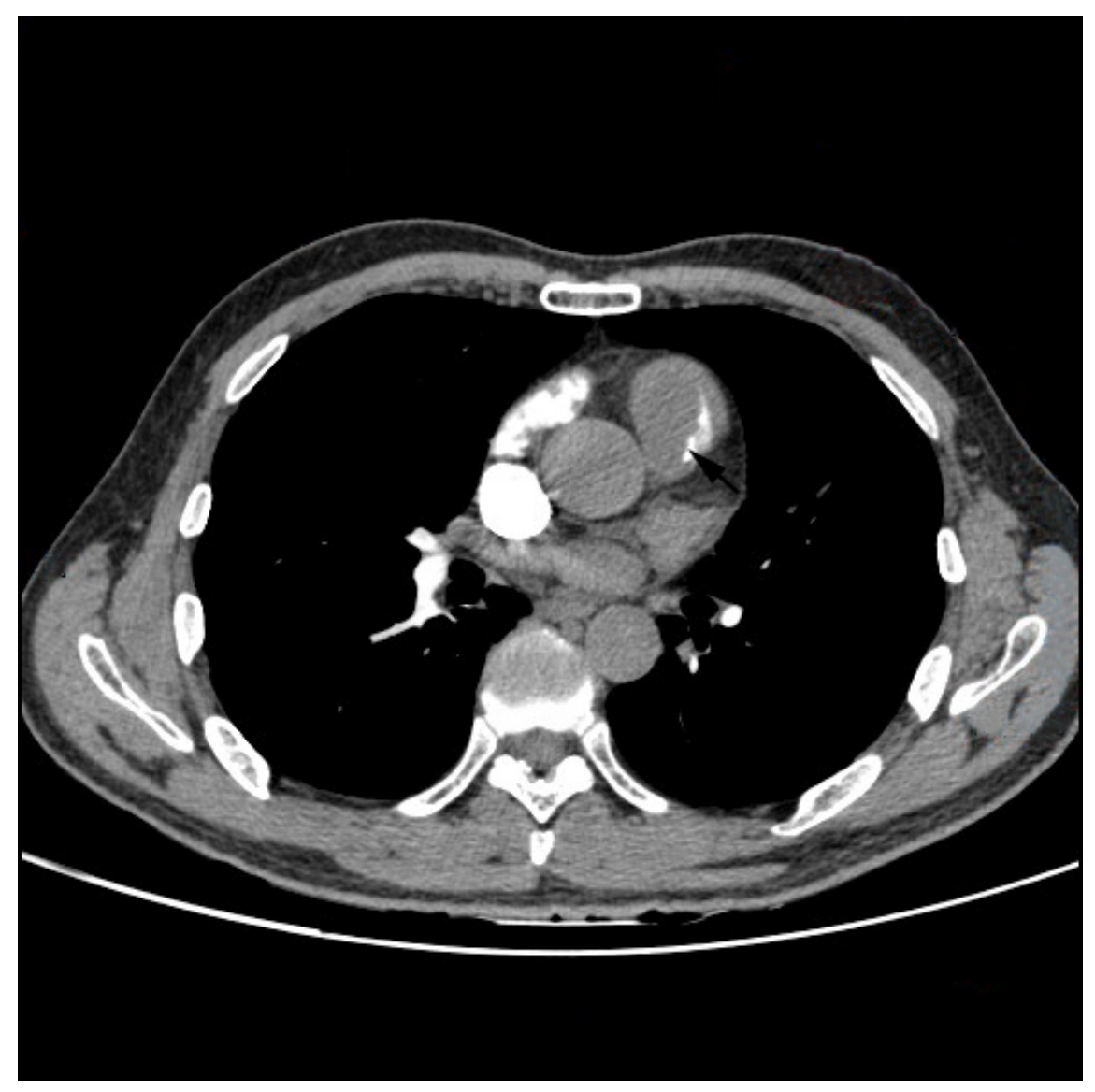



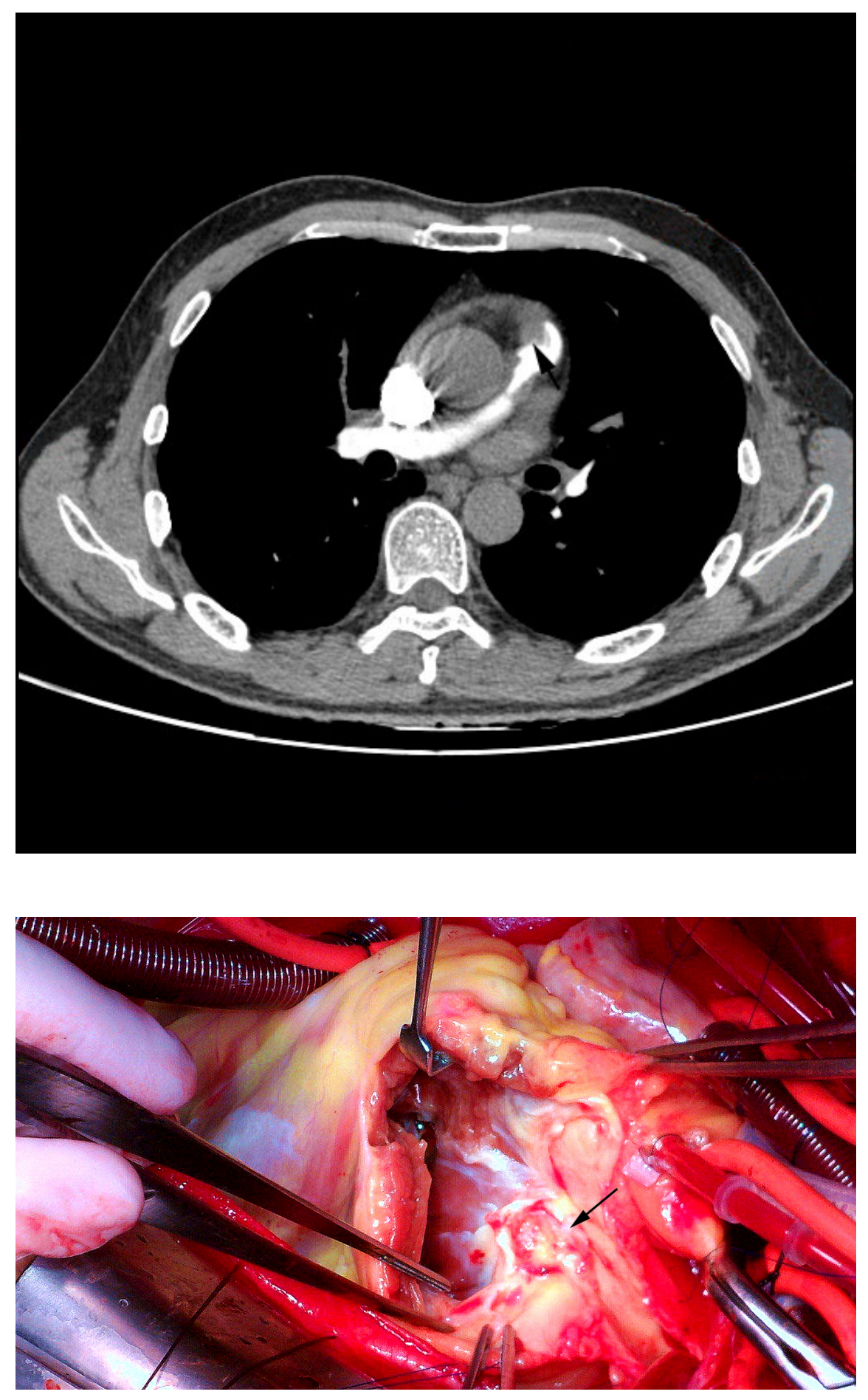

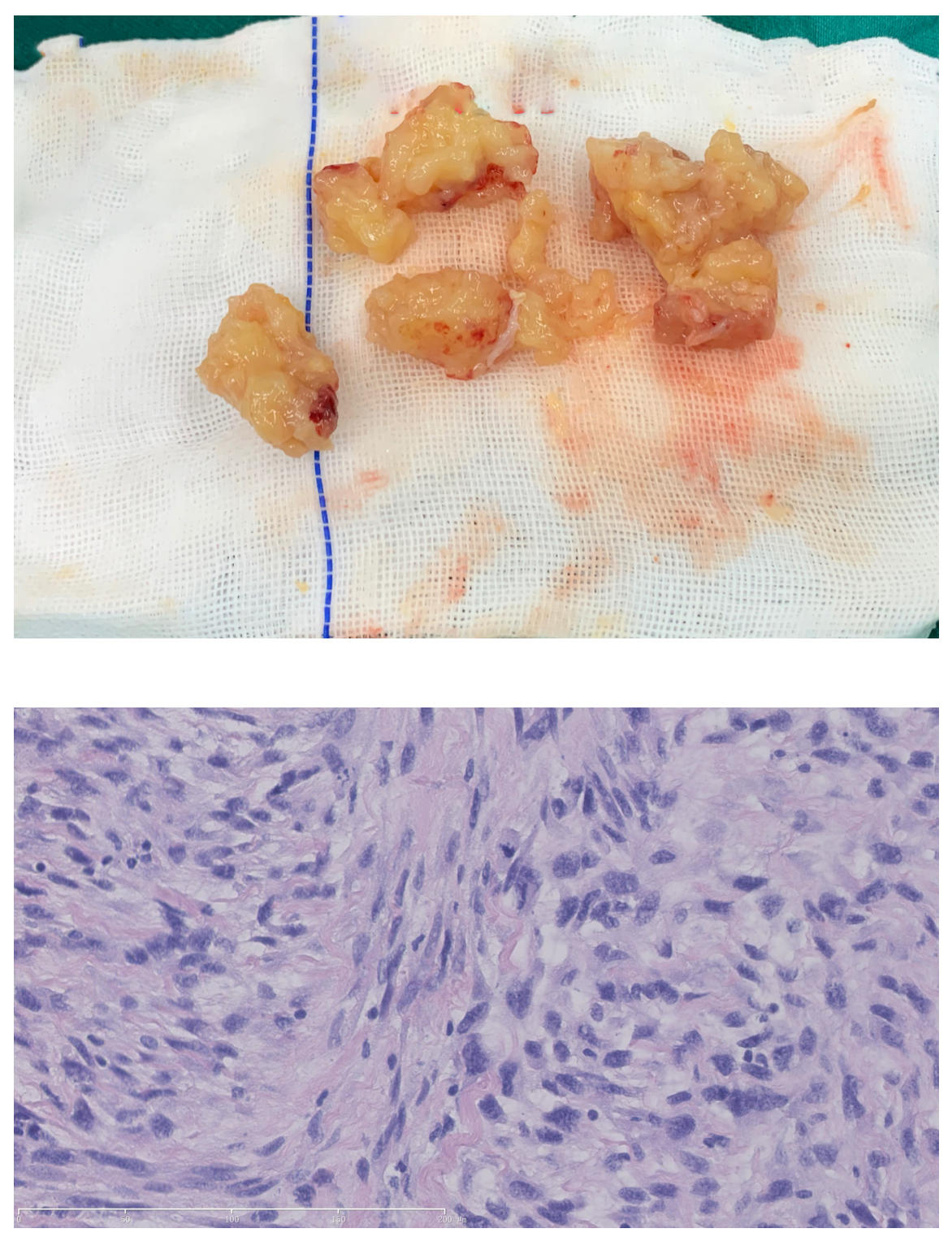